\title{
Correlation of CBD/CHD angulation with recurrent cholangitis in patients treated with ERCP
}

Authors

Institutions
Charing CN Chong ${ }^{1}$, Philip WY Chiu ${ }^{1,2}$, Teresa Tan ${ }^{1}$, Anthony YB Teoh ${ }^{1}$, Kit Fai Lee ${ }^{1}$, Enders Kwok Wai Ng ${ }^{1,2}$, Paul BS Lai ${ }^{1,2}$, James YW Lau ${ }^{1,2}$

${ }^{1}$ Department of Surgery, Prince of Wales Hospital, The Chinese University of Hong Kong

${ }^{2}$ Institute of Digestive Diseases, Prince of Wales Hospital, The Chinese University of Hong Kong

\section{Bibliography}

Dol http://dx.doi.org/

10.1055/s-0035-1569689

Endoscopy International Open

2016; 04: E62-E67

(c) Georg Thieme Verlag KG

Stuttgart · New York

E-ISSN 2196-9736

Corresponding author Chong Ching Ning Charing Surgery

4/F, Clinical Sciences Building

Prince of Wales Hospital,

Shatin, N. T.

Hong Kong, SAR

chongcn@surgery.cuhk.edu.hk
Background and study aims: Endoscopic retrograde cholangiopancreatography (ERCP) with endoscopic sphincterotomy (EST) for bile duct stone extraction has a major role in the treatment of cholangitis. It is well known that certain risk factors predispose to recurrence of such stones. The aims of this study were to evaluate the correlation between angulation of the common bile duct (CBD), right hepatic duct (RHD), and left hepatic duct (LHD) with recurrent cholangitic attacks and to elucidate other risk factors that may be associated with these attacks.

Patients and Methods: This is retrospective study included 62 patients who had undergone therapeutic endoscopic retrograde cholangiopancreatography (ERCP) for bile duct stones. Their medical records were followed until May 1, 2009. The RHD, LHD, and CBD angulation and CBD diameter

\section{Introduction}

$\nabla$

Cholangitis occurs due to acute obstruction of extrahepatic bile ducts with bile stasis, which leads to secondary bacterial infection. In most cases, the obstruction is caused by common bile duct (CBD) or left/right hepatic ductal stones. Therefore, the goal of treatment is to re-establish good biliary drainage and remove stones.

Currently, endoscopic retrograde cholangiopancreatography (ERCP) with endoscopic sphincterotomy (EST) is a well-established therapeutic procedure for treatment of bile duct stones. However, even after first-time endoscopic complete clearance of stones, the rate of recurrence of bile duct stones has been reported to range from $4 \%$ to $24 \%$.

Previous studies have been devoted to eliciting possible risk factors that predispose patients to recurrence of symptomatic stones following EST and stone clearance. One of the important risk factors reported by Keizman et al [1] is acute CBD angulation $\left(\leq 145^{\circ}\right)$. Keizman et al postulated that were measured on cholangiography prior to any endoscopic procedures.

Results: Among these 62 patients, 6 (9.7\%) had recurrence of cholangitis. Both angles of the RHD and the CBD were significantly smaller in the group with recurrence $(P=0.001, P=0.004)$. A CBD angle $\leq 130^{\circ}$ and RHD angle $\leq 125^{\circ}$ were found to be significantly associated with an increased risk of recurrence ( $R R=10.526, P=0.033$; $\mathrm{RR}=24.97, \quad P=0.008$ ) in multivariate analysis. Cholecystectomy was not a protective factor against recurrence of cholangitis $(P=0.615)$. Conclusions: Angulation of the $\operatorname{CBD}\left(\leq 130^{\circ}\right)$ and $\operatorname{RHD}\left(\leq 125^{\circ}\right)$ on ERCP are independent risk factors for recurrent cholangitis. Further prospective studies using these data may be warranted for a more accurate estimation and verification of the risk factors predisposing to recurrent cholangitis.

an angulated CBD would contribute to bile stasis, and hence, is a risk factor for recurrent cholangitis after successful endoscopic therapy. However, no study has yet provided information on the correlation between the angulation of the right/left hepatic ducts (RHD, LHD) and recurrence of cholangitis.

Therefore we performed a retrospective study to examine whether angulation between the RHD, LHD, and CBD is a significant independent predictor of recurrent symptomatic ductal stones. Identification of significant risk factors would allow for follow up of at-risk patients with surveillance ERCP for detection of an asymptomatic stone and prevention of possible recurrence of cholangitis.

\section{Patients and Methods}

$\nabla$

Patients who underwent ERCP for suspected cholangitis in Prince of Wales hospital in 2006 were recruited. Eligible patients were first identified from the 2006 ERCP reports. Their medical notes 


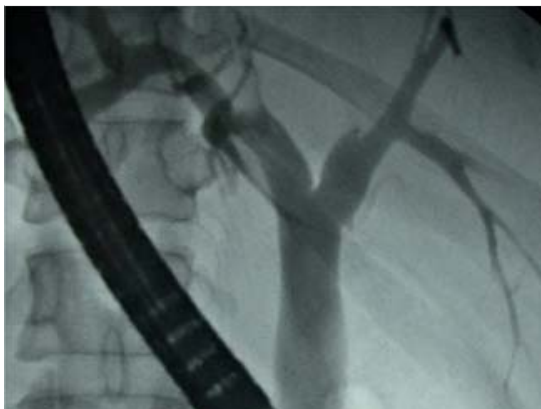

Fig. 1 ERCP film before angle measurement

and ERCP films were subsequently reviewed. A patients was included in the study if: 1 ) he or she presented with symptoms suggestive of cholangitis, including a combination of fever, jaundice, abdominal pain, and had cholestatic biochemical liver function test results; 2) cholangitis was diagnosed on ERCP with either identification of CBD stone or CBD dilatation; and 3) ERCP demonstrated complete removal of initial $\mathrm{CBD}$ stone by clear occlusion cholangiogram (OC). Complete removal of initial CBD stone was an inclusion criterion because it ensured that recurrence was due to new stone formation as opposed to a retained stone.

A patients was excluded if: 1) the attack that occurred in 2006 was not the first episode of cholangitis; 2) he or she underwent previous biliodigestive anastomosis, gastric surgery or CBD exploration, because these surgical procedures could have affected the angulation of the CBD, RHD, and LHD; 3 ) he or she had a known malignancy of the bile ducts, pancreas, or liver or a malignancy diagnosed by brush cytology or biopsy during ERCP because any recurrence of cholangitis would be secondary to obstruction of the CBD by the tumor instead of CBD stone; 4) stenosis, benign or malignant, was the only ERCP finding; 5) ERCP films were unavailable; or 6) CBD, RHD, and LHD were poorly shown after contrast injection.

Patients were followed until December 31, 2011 so that at least 5year follow up could be assured. Recurrence was defined by a combination of clinical symptoms, liver function test, and ERCP. The follow-up end point was defined as either if the patient death or achievement of the end point.

\section{Peri-procedure data}

Each patient's medical data, including age, gender, body mass index (BMI), and smoking and drinking habits were recorded. Results of four liver function tests (LFT) including the first LFT after admission, post ERCP, between post ECRP and discharge and LFT before discharge were recorded.

\section{Endoscopic procedure data}

All ERCPs were performed with a JF 240 or a TJF 240 duodenoscope (Olympus Co., Tokyo, Japan). Information on the endoscopic procedure was retrieved from the endoscopy reports in the endoscopy center. Indications for ERCP were noted. During ERCP, the presence of stone and periampullary diverticulum and the use of mechanical lithotripter (BML) in stone extraction were recorded. All patients included in the study must have had OC performed and complete clearance of stones. Complete sphincterotomy was performed on all patients. Diagnoses on ERCP, such as cholangitis, symptomatic stones, stricture, dilatation, $\mathrm{RPC}$, and normal, were recorded to ensure the inclusion and exclusion of appropriate patients.

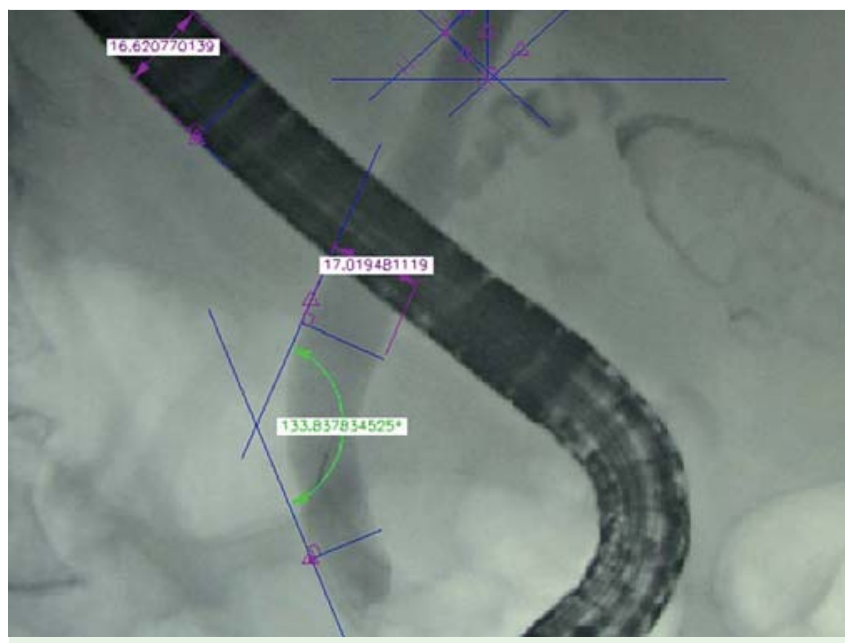

Fig. 2 Measurement of common bile duct angle (CBD). The widest point above and below the angulation of the CBD was identified and perpendicular lines were drawn. The CBD angle was measured from the perpendicular lines (green arrow).

\section{Measurement of bile duct angulation and diameter}

The angulation of the three ducts and diameter of CBD were measured using the pre-procedure $\mathrm{OC}$ with the patient in prone position ( $\bullet$ Fig. 1) Pre-procedure OC was used because withdrawal of the inflated balloon catheter along the $\mathrm{CBD}$ during $\mathrm{OC}$ could have altered the angulation of the ducts. Angulation of CBD was measured using the sharpest angle along CBD from $1 \mathrm{~cm}$ below the bifurcation to $1 \mathrm{~cm}$ above the papilla as described by Keizman et al [1]. The widest point above and below the angulation of the $\mathrm{CBD}$ was identified and perpendicular lines were drawn. The CBD angle was measured from the perpendicular lines ( Fig. 2). Because no reports exist about how to measure RHD and LHD angulation, we devised our own methods. RHD and LHD angulation was defined by the angle between the RHD and CBD, LHD, and CBD, respectively. It was measured by drawing straight lines from the tip of the bifurcation of the CBD to the RHD and LHD, which are perpendicular to the two sides of the RHD and LHD, respectively ( Fig.3a). A line across the CBD around $1 \mathrm{~cm}$ below the bifurcation was also drawn ( $\mathbf{F i g} . \mathbf{3}$ b). Lines perpendicular to these lines were drawn and the angle between the perpendicular lines was measured ( $\bullet$ Fig. $3 \mathrm{c}$ and $\bullet$ Fig. $3 \mathrm{~d}$ ).

The CBD diameter was measured at its widest point, corrected magnification relative to the known diameter of the endoscope. The actual CBD diameter was calculated using this formula: (measured CBD diameter/measured endoscope diameter) $\times$ actual diameter of endoscopic $(11 \mathrm{~mm})$. All ERCP conducted in 2006 were performed using the same type of endoscopic with the same diameter. All measurements were performed using computer freeware PTC ProDESKTOP 2000i2. The cholangiogram analysis was performed by two investigators, who were blinded to the outcome of the patients.

\section{Statistical analysis}

Categorical variables (gender, smoking, drinking, periampullary diverticulum, and mechanical lithotripter) were compared using Pearson's Chi square test or Fisher's exact test when both variables were dichotomous. Continuous variables (age, BMI, liver function test, CBD diameter, and CBD, RHD, and LHD angulation) were analyzed using the Student's $t$ test when the variables were 

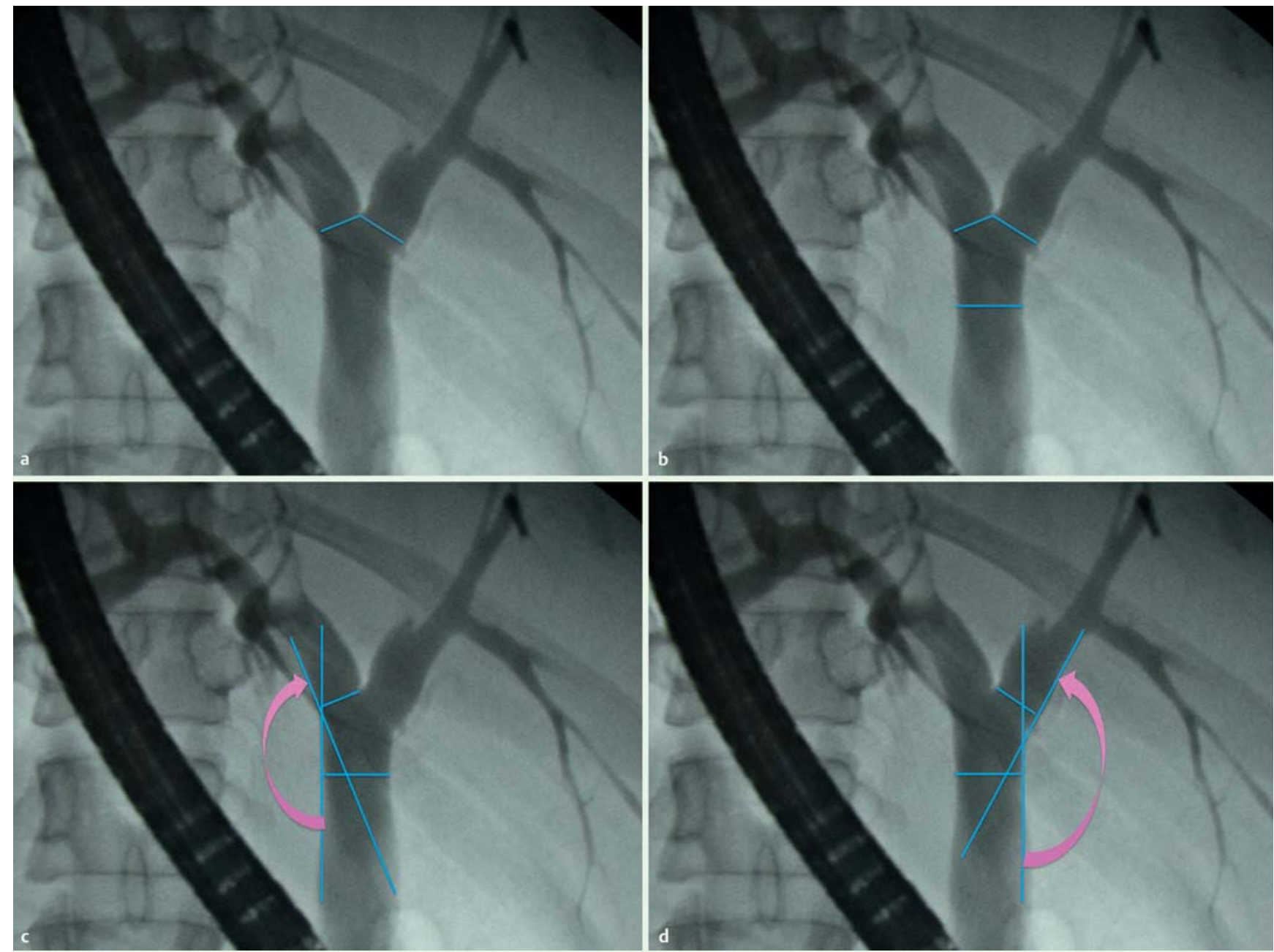

Fig.3 Measurement of right and left hepatic duct angle. a Straight lines were drawn from the tip of the bifurcation of the common bile duct (CBD) to the right hepatic duct (RHD) and left hepatic (LHD), which are perpendicular to the sides of the RHD and LHD respectively. $\mathbf{b}$ A line across the CBD around $1 \mathrm{~cm}$ below the bifurcation was also drawn. c Measurement of RHD angle. Line perpendicular to the lines described in $\mathbf{a}$ and $\mathbf{b}$ were drawn. The RHD angle was measured between the perpendicular lines (pink arrow). $\mathbf{d}$ Measurement of LHD angle. Lines perpendicular to the lines described in $\mathbf{a}$ and $\mathbf{b}$ were drawn. The LHD angle was measured between the perpendicular lines (pink arrow).

normally distributed or by Mann Whitney U test for variables without normal distribution.

Significant predictors for bile duct stone recurrence identified by univariate analysis were included in a multiple logistic regression model to determine the most significant risk factors for recurrence of cholangitis. A $P$ value $\leq 0.05$ was considered statistically significant and all tests were two-sided. Statistical analysis was conducted using the SPSS package 15.

\section{Results}

$\nabla$

In 2006, a total of 463 patients underwent ERCP in the Prince of Wales Hospital. Among them, 62 patients who fulfilled the inclusion and exclusion criteria were recruited into our study. The mean age of the patients was 68.5 years and $28(45.2 \%)$ were male. Of the patients included in our study, six suffered from minor bleeding during ERCP, which was controlled with adrenaline spray. One developed hypotension but the ERCP was completed. None of the patients suffered from perforation or post-ERCP pancreatitis. The mean CBD diameter on index ERCP was $17.2 \mathrm{~mm}$.
Of the 62 patients, 48 (77.4\%) had presence of CBD stones identified on the index ERCP. The size of CBD stones ranged from 3 to $30 \mathrm{~mm}$ and multiple stones were identified in 10 cases. CBD sludge was found and removed in five cases. In the remaining nine patients, the clinical picture was compatible with passed stones as gallstones were demonstrated on transabdominal ultrasound or computed tomography before ERCP and clinical improvement was evident after ERCP. As a result, these patients were also included in this study.

Six of the patients $(9.7 \%)$ had recurrence of cholangitis and 55 (90.2\%) had no recurrence as of December 31, 2011, which was the end point of our follow-up. Of the six patients with recurrence, all except one had recurrence of CBD stones at distal CBD. The remaining patient had CBD stone recurrence at mid-CBD. The median follow-up period was 62.7 months (range: 5.3 months to 71.7 months). The demographic data on the two groups, including age, gender and BMI, were comparable ( $\bullet$ Table 1). No significant difference was seen between the two groups in the number of smokers and drinkers.

Stone recurrence due to migration from the gallbladder might be a possibility. Therefore, we reviewed the gallbladder status of the patients. In total, 12 (19.7\%) patients received cholecystectomy, 


\begin{tabular}{|c|c|c|c|}
\hline \multirow[b]{2}{*}{ Factors } & \multicolumn{2}{|c|}{ Recurrent attack of cholangitis } & \multirow[t]{2}{*}{$P$ value } \\
\hline & Yes & No & \\
\hline Gender & & & 0.150 \\
\hline Male & 4 & 2 & \\
\hline Female & 2 & 31 & \\
\hline Smoker & 4 & 17 & 0.160 \\
\hline Drinker & 3 & 7 & 0.073 \\
\hline Periampullary diverticula & 1 & 20 & 0.227 \\
\hline Basket Mechanical lithotripsy & 1 & 7 & 0.647 \\
\hline Gallbladder stone & 4 & 16 & 0.151 \\
\hline Cholecystectomy & 2 & 10 & 0.418 \\
\hline BMI (mean) & 23.424 & 24.124 & 0.706 \\
\hline Age (mean) & 62.439 & 68.889 & 0.332 \\
\hline Bilirubin (after admission) & 88.286 & 89.415 & 0.702 \\
\hline ALT (after admission) & 241.571 & 281.981 & 0.365 \\
\hline ALP (after admission) & 162.286 & 249.396 & 0.194 \\
\hline Bilirubin (post-ERCP) & 88.000 & 71.122 & 0.918 \\
\hline ALT (post-ERCP) & 250.000 & 180.122 & 0.945 \\
\hline ALP (post-ERCP) & 173.600 & 207.781 & 0.560 \\
\hline Bilirubin (in between) & 88.500 & 48.920 & 0.570 \\
\hline ALT (in between) & 67.000 & 120.280 & 0.462 \\
\hline ALP (in between) & 185.500 & 166.440 & 1.000 \\
\hline Bilirubin (before discharge) & 46.333 & 33.024 & 0.620 \\
\hline ALT (before discharge) & 54.333 & 98.952 & 0.259 \\
\hline ALP (before discharge) & 111.666 & 170.071 & 0.259 \\
\hline CBD diameter & 11.411 & 11.555 & 0.886 \\
\hline Common bile duct angle & 131.845 & 148.221 & 0.002 \\
\hline Left hepatic duct angle & 140.999 & 139.096 & 0.938 \\
\hline Right hepatic duct angle & 122.739 & 138.623 & 0.000 \\
\hline
\end{tabular}

Table 1 Univariate analysis for recurrent attack of cholangitis.

BMI: body mass index; ALT, alanine transferase; ALP, alkaline phosphatase; ERCP, endoscopic retrograde cholangiopancreatography CBD, common bile duct

of whom 2 were in the recurrence group. Cholecystectomy was not a protective factor against recurrence of cholangitis $(P=$ 0.615). We also looked at three other factors, including stone extraction using mechanical lithotripter basket, presence of periampullary diverticulum, and gallstones. Twenty-one (34.4\%) patients had periampullary diverticulum, 20 (32.8\%) had a history of gallstones, and eight (13.1\%) received mechanical lithotripsy. No significant difference was seen between the two groups in any of the three factors ( $P=0.405,0.203,1.000$ respectively).

Regarding the angulation of CBD and RHD, they were significantly smaller in the group with recurrence than in the non-recurrent group ( $P=0.001$ and 0.004 , respectively) in univariate analysis. For the RHD, an angulation $\leq 125^{\circ}$ was significantly associated with an increased risk of recurrence ( $R R=24.97,95 \%$ C.I. $=$ 2.276-274.014, $P=0.008$ ) in multivariate analysis ( Table 2 ). The association was similar for CBD with an angulation $\leq 130^{\circ}$ $(\mathrm{RR}=10.526,95 \%$ C. $\mathrm{I} .=1.204-92.012, P=0.033)$ in multivariate analysis ( Table 2). LHD angulation was not associated with recurrence $(P=0.928)$. There was also no significant difference between the two groups in CBD diameter $(P=0.886)$.

\section{Discussion}

\section{$\nabla$}

In this study, we identified two independent risk factors for symptomatic bile duct stone recurrence after successful endoscopic therapy: 1) CBD angulation $\left(\leq 130^{\circ}\right)$; and 2) RHD angulation $\left(\leq 125^{\circ}\right)$. Of these two findings, CBD angulation has been associated with stone recurrence in a previous study by Keizman
Table 2 Independent risk factors for recurrent bile duct stones in multivariate analysis.

\begin{tabular}{|llll|}
\hline Factor & Relative risk & $\mathbf{9 5 \%} \mathbf{C l}$ & $\boldsymbol{P}$ value \\
\hline $\mathrm{CBD}$ angle $\leq 130^{\circ}$ & 10.526 & $1.204-92.012$ & 0.033 \\
\hline RHD angle $\leq 125^{\circ}$ & 24.97 & $2.276-274.014$ & 0.008 \\
\hline
\end{tabular}

CBD, common bile duct; RHD, right hepatic duct

et al, [1] whereas an association with RHD angulation has not been previously reported in the literature.

Establishing an appropriate follow-up time is crucial to the design of this study as a sufficient period of follow-up time is needed for recurrence to occur so that statistical analysis can be performed. In our current study, the mean follow-up time for our patients was 62.7 months (>5 years). Earlier studies have shown that most recurrences occurred within the first 2 years after the first ERCP $[2,3]$. Other long-term follow-up studies performed also found that the peak time of recurrence is 2 to 3 years after the first attack $[4,5]$. Therefore, we believe that we have allowed sufficient follow-up time. Moreover, we observed a recurrence rate of $13 \%$, which is comparable to that in most studies conducted [6-9].

Warren [10] first described an association between acute CBD angulation and cholangitis compared to patients with cholecystitis during open surgery. A similar study conducted by Keizman et al [1] also found $\mathrm{CBD}$ angulation of $\leq 145^{\circ}$ to be an independent risk factor for recurrence of cholangitis. Indeed, angulation along the course of the $\mathrm{CBD}$ promotes bile stasis and may predispose to stone formation and recurrence. However, we found a much 
smaller CBD angulation, $\leq 130^{\circ}$, to be related to recurrent cholangitis. The findings by Keizmen et al [1] may have differed because of use of post-procedural ERCP film. The inflated balloon pulled through the CBD during occlusion cholangiogram could have straightened the $\mathrm{CBD}$ and increased the angulation.

To the best of our knowledge, this study is the only one to describe the association between RHD and LHD angulation and recurrence of cholangitis. We are also the first to discover the relationship between sharp RHD angulation $\left(\leq 125^{\circ}\right)$ with recurrent cholangitis. Jeng et al described the association between sharp angulation $\left(<90^{\circ}\right)$ and worse surgical treatment outcomes in patients with recurrent pyogenic cholangitis (RPC) [11]. However, none of the patients in our study had an RHD angle $<90^{\circ}$. This may be due to differences inherent in our study population. The study by Jeng et al was performed in a referral center in which surgical treatment was being planned for the patients. Therefore, his patients with RHD angulation $<90^{\circ}$ may represent those with more severe disease requiring surgery.

Our findings have important clinical relevance. Recurrent cholangitis can be life-threatening because of the potential for repeated infections and systemic sepsis. Therefore, it is important that an effort be made to identify patients at high risk of recurrence to permit close follow-up evaluation. Surveillance ERCP has been shown to decrease the average number of cholangitis episodes from 2 to 0.6 per patient within a 4-year period [4]. It has also been shown to detect asymptomatic recurrences that would have otherwise led to another episode of ascending cholangitis $[4,8]$ and to reduce mortality [6]. Geenen et al [4] thus proposes conducting surveillance ERCP annually for approximately 4 years. However, ERCP can be uncomfortable for patients, invasive, and expensive. In the modern era, the role of purely diagnostic ERCP is being replaced by MRCP. We thus propose that patients with a combination of risk factors, angulated $\operatorname{CBD}\left(\leq 130^{\circ}\right)$, angulated RHD $\left(\leq 125^{\circ}\right)$, and dilated $\mathrm{CBD}$ undergo annual surveillance MRCP.

Whether surgical intervention is a good alternative remains a complex question. Various treatment options should be considered, including liver resection, biliary drainage alone, combined liver resection and biliary drainage, and percutaneous choledochoscopy. Biliary drainage is usually performed as a hepaticojejunostomy with or without the creation of an access loop to facilitate the subsequent retrieval of recurrent stones. Other less commonly used drainage procedures include transduodenal sphincteroplasty or T-tube drainage. The choice of therapy has to be decided based on a patient's condition, such as fitness for general anesthesia, tolerance for hepatectomy, previous treatment failure, multiple previous operations, and local anatomic factors [12]. Surgery may only be suitable for a limited number of patients who are younger and can tolerate a procedure, or who have multiple recurrence, multiple stones, or dilatation of duct or presence of stricture $[4-5,13]$.

Interestingly, there may be multiple reasons why LHD angulation was not related to recurrent cholangitis in our study. First, unlike RHD, LHD may not be related to recurrent cholangitis. Second, our sample size may have been inadequate to detect an association, because there were only seven cases of recurrence. Similarly, the sample size may also have been inadequate to detect a significant difference in the CBD diameter, because the majority of previous studies found an association between greater CBD diameter and recurrent cholangitis $[1,6,7,9]$.

Routine USG of the hepatobiliary system is not normally performed on all patients who present with acute cholangitis. In- stead, they are booked for an early ERCP. Therefore, gallstones can only be found if USG is selectively performed or alternatively, if the gallbladder is filled with contrast on ERCP. As a result, in this retrospective study, we do not know the true rate of patients with gallstones in our study population and, therefore, the number mostly likely is an underestimation. Given that, we cannot draw a firm conclusion about whether gallstones are a risk factor for recurrent cholangitis. Furthermore, elective cholecystectomy was found to be ineffective in reducing the incidence of recurrent cholangitis. This finding is consistent with other studies $[1,7,9$, 14]. In contrast to the West, in Asian countries, the incidence of primary biliary stones may be relatively higher compared to the number of secondary CBD stones originating from the gallbladder. [15] This may be the reason why elective cholecystectomy is not effective in preventing recurrent cholangitis in an Asian population.

Peripapillary diverticulum is one of the reported potential causes of recurrent CBD stones. However, conclusions about whether periampullary diverticulum leads to recurrent cholangitis are still contradictory [16-19]. A few studies have found it to be a risk factor for choledocholithiasis, $[6,15]$ but others have not $[1$, $7,14,20]$. In the current study, we did not find periampullary diverticulum to be a risk factor for recurrent cholangitis. Results of all four liver function tests performed and recorded at different times during admission were not found to be associated with recurrence, which is similar to findings by Costamagna et al [7]. Our study found that BML is not a risk factor for recurrence, as did a study conducted by Costamagna et al, but Saito et al [8] showed otherwise. Costamagna et al argued that because Satio et al did not perform complete sphincterotomy, spontaneous passage of the minute stone fragments could not have occurred, leading to the false impression that BML is a risk factor for stone recurrence. Because all the patients in our study had complete sphincterotomy and achieved complete CBD clearance on OC, BML was not found to be a risk factor for recurrence.

There are some limitations to our study. First, small sample size is one of the major flaws of our paper. Second, this study deals only with symptomatic recurrence of stones, and so, our cases were diagnosed based on clinical grounds, backed by laboratory, ultrasonographic and/or ERCP evidence. Therefore, there may have been false negatives in view of the failure to detect the asymptomatic recurrence of stones. The recurrence reported above might represent an underestimation. Third, the measurement of the angulation between the intrahepatic ducts and the common bile ducts might not be exact, given that the calculation was done on patients in the prone position and that bile angulation was assessed in a two-dimensional plane. That may have led to an underor overestimation of CBD, RHD or LHD angulation. We tried to ensure that the calculation was as accurate as possible by standardizing the method of measuring and using only non-rotated pre-procedure cholangiograms with patients in a prone position. In future studies, magnetic resonant cholangiopancreatogram (MRCP) could be used to assess the angulation in a three- dimensional plane to increase accuracy of angle assessment. Last, measuring bile duct angles does not seem easy. It is hoped that, as technology advances and through collaboration with engineers, it may be feasible to develop new software or apps to make measurement easier in daily practice. 


\section{Conclusions}

$\nabla$

An angulated CBD and RHD are risk factors for recurrent cholangitis. This finding can assist us in identifying patients who may be at risk. Annual MRCP surveillance of this group of patients has the potential to reduce the morbidity and mortality associated with an acute attack. Future prospective studies with 3D imaging may be needed to further validate the association between bile duct angles and recurrent cholangitis.

\section{Competing interests: None}

\section{Acknowledgements \\ $\nabla$}

The authors thank Cindy Li, Vivianne Luk, Alexandra Ng, Karen Tand, and June Wong for their work on the bile duct angle measurement.

\section{References}

1 Keizman D, Shalom MI, Konikoff FM. An angulated common bile duct predisposes to recurrent symptomatic bile duct stones after endoscopic stone extraction. Surg Endosc 2006; 20: 1594-1599

2 Jacobsen 0 , Matzen P. Long term follow up study of patients after endoscopic sphincterotomy for choledocholithiasis. Scan J Gastroenterol 1987; 22: 903 - 906

3 Kullman E, Borch K, Liedberg G. Long term follow up after endoscopic management of retained and recurrent common duct stones. Acta Chir Scand 1989; 155: 395 - 399

4 Geenen DJ, Geenen FM, Jafri FM et al. The role of surveillance endoscopic retrograde cholangiopancreatography in preventing episodic cholangitis in patients with recurrent common bile duct stones. Endoscopy 1998; 30: 18-20

5 Sato T, Suzuki N, Takahashi W. Follow up studies of surgical treatment of choledocholithiasis. Toboku J Exp Med 1981; 135: 1 -9

6 Pereira Lima JC, Jakobs R, Winter UH et al. Long term results (7 to 10 years) of endoscopic papillotomy for choledocholithiasis. Multivariate analysis of prognostic factors for the recurrence of biliary symptoms. Gastrointest Endosc 1998; 48: 457-464

7 Costamagna $G$, Tringali A, Shah SK et al. Long term follow up of patients after endoscopic sphincterotomy for choledocholithiasis, and risk factors for recurrence. Endoscopy 2002; 34: 273 - 279

8 Satio M, Tsuyuguchi T, Yamaguchi T et al. Long term outcome of endoscopic papillotomy for choledocholithiasis with cholecystolithiasis. Gastrointest Endosc 2000; 51: 540-545.9

9 Ueno N, Ozawa Y, Aizawa T. Prognostic factors for recurrence of bile duct stones after endoscopic treatment by sphincter dilation. Gatrointest Endosc 2003; 58: 336 - 340

10 Warren BL. Association between cholangiographic angulation of the common bile duct and choledocholithiasis. S Afr J Surg 1987; 25: 13 - 5

11 Jeng KS, Ohta I, Yang FS et al. Coexisting sharp ductal angulation with intrahepatic biliary strictures in right hepatolithiasis. Arch Surg 1994; 129: 1097 - 1102

12 Lee $\mathrm{KF}$, Chong $\mathrm{CN}, \mathrm{Ng} \mathrm{D}$ et al. Outcome of surgical treatment for recurrent pyogenic cholangitis: a single-centre study. HPB (Oxford). PubMed PMID 19590627; PubMed Central PMCID: PMC2697866 02 2009; 11: 75 - 80 (DOI 10.1111/j.1477-2574.2008.00018.x)

13 Bergman JJGHM, van der Mey S, Rauws EAJ et al. Long term follow up after endoscopic sphincterotomy for bile duct stones in patients younger than 60 years of age. Gastrointest Endosc 1996: 44; 643 - 649

14 Lai $K H$, Lin LF, Lo GH et al. Does cholecystectomy after endoscopic sphincterotomy prevent the recurrence of biliary complications? Gastrointest Endosc 1999; 49: $483-487$

15 Ando T, Tsuyuguchi T, Okugawa $T$ et al. Risk factor for recurrent bile duct stones after endoscopic papillotomy. Gut 2003; 52: 116-121

16 Kim MH, Myung SJ, Seo DW et al. Association of periampullary diverticula with primary choledocholithiasis but not with secondary choledocholithiasis. Endoscopy 1998; 30: 601 - 604

17 Chandy G, Hart WJ, Roberts-Thomson IC. An analysis of the relationship between bile duct stones and periampullary duodenal diverticula. J Gastroenterol Hepatol 1997; 12: 29-33

18 Hall RI, Ingoldby CJ, Denyer ME. Periampullary diverticula predispose to primary rather than secondary stones in the common bile duct Endoscopy 1990; 22: 127-128

19 Kennedy RH, Thompson $M H$. Are duodenal diverticula associated with choledocholithiasis? Gut 1988; 29: 1003-1006

20 Hammatstrom LE, Stridbeck H, Ihse I. Long term follow up after endoscopic treatment of bile duct calculi in cholecystectomized patients. World J. Surg 1996; 20: 272-276 